\title{
Endovascular repair of ascending aorta pseudoaneurysm
}

\author{
Luca Di Marco ${ }^{1}$, Luigi Lovato ${ }^{2}$, Giacomo Murana ${ }^{1}$, Ciro Amodio ${ }^{1}$, Francesco Buia ${ }^{2}$, Roberto Di \\ Bartolomeo $^{1}$, Davide Pacini ${ }^{1}$ \\ ${ }^{1}$ Cardiac Surgery Unit, ${ }^{2}$ Radiology Unit, Cardio-Thoracic-Vascular Department, S. Orsola Hospital, University of Bologna, Bologna, Italy \\ Correspondence to: Luca Di Marco, MD, PhD. Sant'Orsola Hospital, Bologna University, via Massarenti 9, 40138 Bologna, Italy. \\ Email: ludima08@libero.it.
}

\begin{abstract}
We present a case of a 48-year-old female patient with Marfan syndrome and previous open surgeries for Bentall procedure and arch replacement with frozen elephant trunk (FET) technique, who was admitted at our Department with a diagnosis of ascending aorta pseudoaneurysm at the anastomosissite between composite valve graft and arch prosthesis treated by endovascular procedure because of she was considered to be at high-risk for a third open surgery and for the patient's favorable anatomy for endovascular closure of the pseudoaneurysm. The patient was successfully treated with the positioning of two abdominal cuffs through the left axillary artery. The postoperative course was uneventful and at 2-month follow-up, the patient was free of complications with CT angiogram showing complete endovascular exclusion of the pseudoaneurysm.
\end{abstract}

Keywords: Endovascular; ascending aorta; stent graft

Received: 30 March 2018; Accepted: 08 May 2018.

doi: $10.21037 /$ jovs.2018.05.15

View this article at: http://dx.doi.org/10.21037/jovs.2018.05.15

\section{Introduction}

Ascending aorta diseases are traditionally treated by surgical replacement. In the last decades, the development and the increasing improvement of the endovascular techniques allowed to treat, above all in high-risk cases, thoracic aortic lesions with stent-graft placement avoiding open surgery (1-3). If this is true, however, for the aortic arch, for the descending thoracic aorta and for the abdominal aorta, it is not so obvious for the ascending aorta whose endovascular treatment continues to be considered a formidable challenge. In fact, due to its complex anatomy, hemodynamic environment, coronary ostia, the endovascular approach for the proximal aorta can be considered the last frontier of the evolution of endovascular aortic therapies, considering that the endovascular aortic arch procedures using branched or fenestrated endografts or open arch debranching, although technically challenging and anatomically treacherous, have proven to be feasible $(1,3,4)$. However, despite the above challenges and difficulty, the ever-increasing experience and the technical evolution during the last years have made it possible to start treating ascending aorta pathologies, including dissections, chronic aneurysms, pseudoaneurysms, penetrating aortic ulcers and intramural hematomas, by the use of endovascular stentgraft (in light of this, several authors have published their experience with endovascular treatment of the ascending aorta with a great variety of indications (5).

\section{Case presentation}

We present a case of a 48-year-old female with Marfan syndrome, pectus excavatum, atrial fibrillation under oral anticoagulation therapy and family history of cardiovascular diseases who was admitted to our department with a diagnosis of ascending aorta pseudoaneurysm.

Her history started in 1999 when she was operated on for type A acute aortic dissection with mechanical composite valve graft according with modified Bentall procedure. From that period, she underwent to a follow up program by computed tomography (CT) angiogram and in 2007 she underwent redo operation of aortic arch replacement with frozen elephant trunk (FET) procedure for residual chronic dissection with the use of E-vita open hybrid stent- 


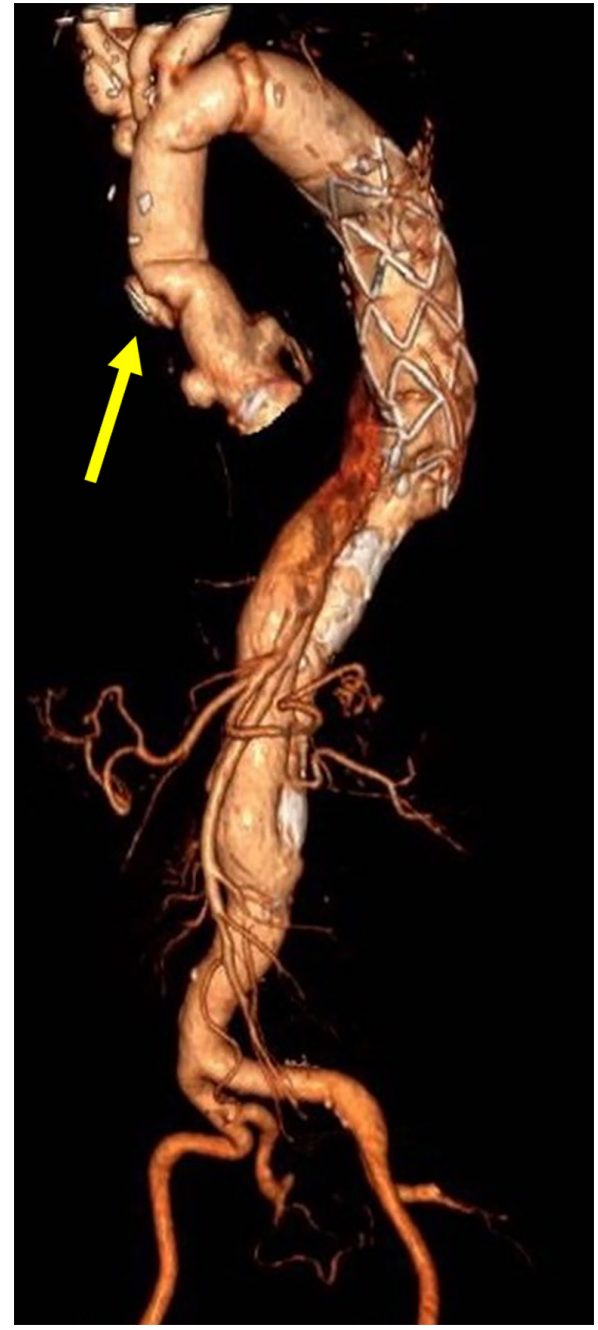

Figure 1 Preoperative $3 \mathrm{D}$ CT angiogram with the pseudoaneurysm (arrow). CT, computed tomography. graft (JOTEC GmbH, Hechingen, Germany). This was the first case of FET we did in our centre and the stent graft was incorrectly placed in the false lumen because of we did not use the guide wire, that we commonly position through the femoral artery in the thoracic aorta, under transesophageal control and after systemic heparinization, in order to be sure to deploy the prosthesis in the true lumen. Fortunately, the presence of many reentries in the downstream aorta allowed the perfusion of both lumens, so that the patient remains stable in the following years. From 2007 the patient remained in a yearly follow-up (FU) program by $\mathrm{CT}$ angiogram which showed a residual aortic dissection distal to E-vita endoprosthesis, with perfusion of both lumens. During the following years, due to a dehiscence of the surgical suture in the ascending aorta, the patient developed a pseudoaneurysm at the surgical anastomosis site between the E-vita prosthesis and the composite valve graft (Figure 1), reason for which, in 2014, an attempt to embolize the pseudoaneurysmatic pouch was made but, due to an aortographic finding of a dimensional reduction of the pseudoaneurysm, it was not performed. For this reason, the patient continued to be monitored with CT angiogram and transthoracic echocardiogram at our outpatient clinic in order to evaluate any dimensional increases of the pseudoaneurysm. The last CT angiogram performed 3 years later the attempt of embolization, showed a $3-\mathrm{mm}$ dimensional increase (from 41 to $44 \mathrm{~mm}$ ) of the pseudoaneurysm. In the light of this dimensional increase, and because of a close contact of the pseudoaneurysm with the sternum for the pectus excavatum, indication to treat the pseudoaneurysm was given (Figure 2). As the aortic team (cardiac surgeons and interventional radiologists) deemed
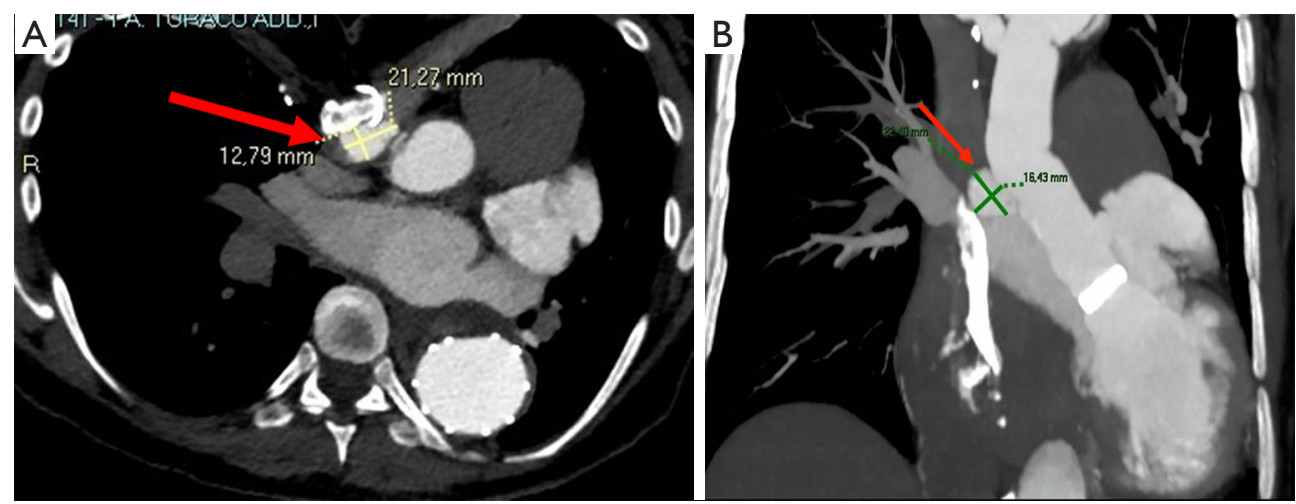

Figure 2 Preoperative CT angiogram shows the close contact between the pseudoaneurysm and the sternum (A) (arrow) and its location at the anastomosis site between the two prostheses (B) (arrow). CT, computed tomography. 


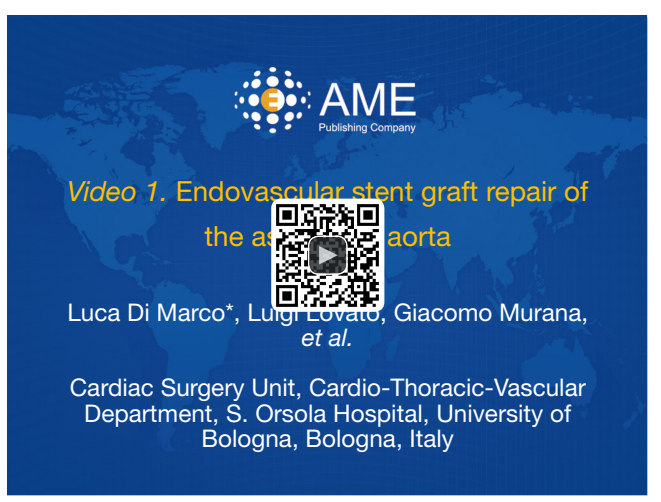

Figure 3 Endovascular stent graft repair of the ascending aorta (6). Available online: http://www.asvide.com/article/view/24982

the patient as high-surgical risk and in order to avoid the third surgical procedure and considering the anatomical features of the pseudoaneurysm, the case seemed suitable for endovascular closure of the pseudoaneurysm. The patient was taken to the hybrid operating room where a transesophageal echocardiogram (TEE) was performed. The TEE confirmed the pseudoaneurysm at the site of surgical-prosthesis to prosthesis-anastomosis. In addition, the TEE showed the mechanical aortic valve prosthesis to be competent without evidence of aortic valve insufficiency. Access to the ascending aorta prosthesis was gained through a left sub-clavicular incision in order to reach the left axillary artery. Vascular access to the artery was obtained through direct puncture of the artery. At the same time, a 5-French pigtail was introduced into the left femoral artery for pre- and post-procedural angiography and positioned at the level of the E-vita prosthesis into the aortic arch. The pre-procedural angiography confirmed the presence of the pseudoaneurysm at the junction between the two ascending aorta vascular prostheses. Through a 6-French introducer positioned into the left axillary artery, a guidewire was positioned into the ascending aorta. Under TEE and fluoroscopic control, the wire was exchanged for the Amplatz super stiff with floppy tip and positioned just above the aortic valve prosthesis. An 18-French Dry Seal Gore introducer was then exchanged and positioned into the aortic arch. Subsequently, a first proximal $4.5-\mathrm{cm}$ abdominal cuff (GORE PLA360400) was released under rapid ventricular pacing (heart rate: $120 \mathrm{bpm}$ ) and controlled hypotension [arterial blood pressure (ABP): $40 \mathrm{mmHg}$ ] into the ascending aorta prosthesis at the site of the pseudoaneurysm. For a better distal sealing we decided to deploy a second $4.5-\mathrm{cm}$ abdominal cuff in the same way, with the proximal portion of the cuff into the first one. TEE and angiography were used to help identify the proximal and distal landing zone for the stent graft and to see the exact position of the coronary ostia. The patient's blood pressure was quickly restored after aortic stent-graft deployment (Figure 3).

Post-procedural angiography showed complete exclusion of the pseudoaneurysm, the absence of any leakage and the patency of the coronary ostia. An intraoperative TEE showed that the aortic valve prosthesis was competent and there were no defects throughout the entire composite valve graft and the Dacron portion of the E-vita prosthesis. The TEE also confirmed the patency of both coronary ostia. The Dry Seal Gore introducer was then removed, the left axillary artery was then clamped proximally and distally and closed by a continuous $6 / 0$ polypropylene suture. The patient remained hemodynamically stable during the whole procedure and was extubated 3 hours postoperatively. Postoperative recovery was uneventful and the patient was discharged home on postoperative day 6 . A pre-discharged CT angiogram confirmed successful endovascular complete exclusion of the pseudoaneurysm of the ascending aorta prostheses (Figure 4). At 2-month follow-up, the patient continued to remain free of complications with CT angiogram showing stable endovascular repair of the pseudoaneurysm (Figure 5).

\section{Discussion}

Endovascular repair of ascending aorta pathologies can be considered, to all effects, the "last bulwark" to be overcome in the field of endovascular treatment of aortic diseases and a formidable challenge in stent graft placement. Despite, in most of cases, ascending aorta endovascular repair is currently reserved to high surgical risk patients with lesions limited to the sinotubular junction or to the tubular portion of the ascending aorta, it is conceivable that, in the immediate future, the endovascular treatment of the ascending aorta may no longer represent the exception but the rule. Actually, a number of different endovascular technologies and approaches have been used, although no consensus exists regarding a standardized technique. In fact, the first attempts of endovascular treatment of ascending aorta pathologies date back to 2000, when Dorros et al. treated a case of acute type A aortic dissection with a custom-made stent graft, complicated by a new type B dissection and patient's death 3 weeks later the procedure for myocardial infarction (7). From 2000 onwards, the cases 

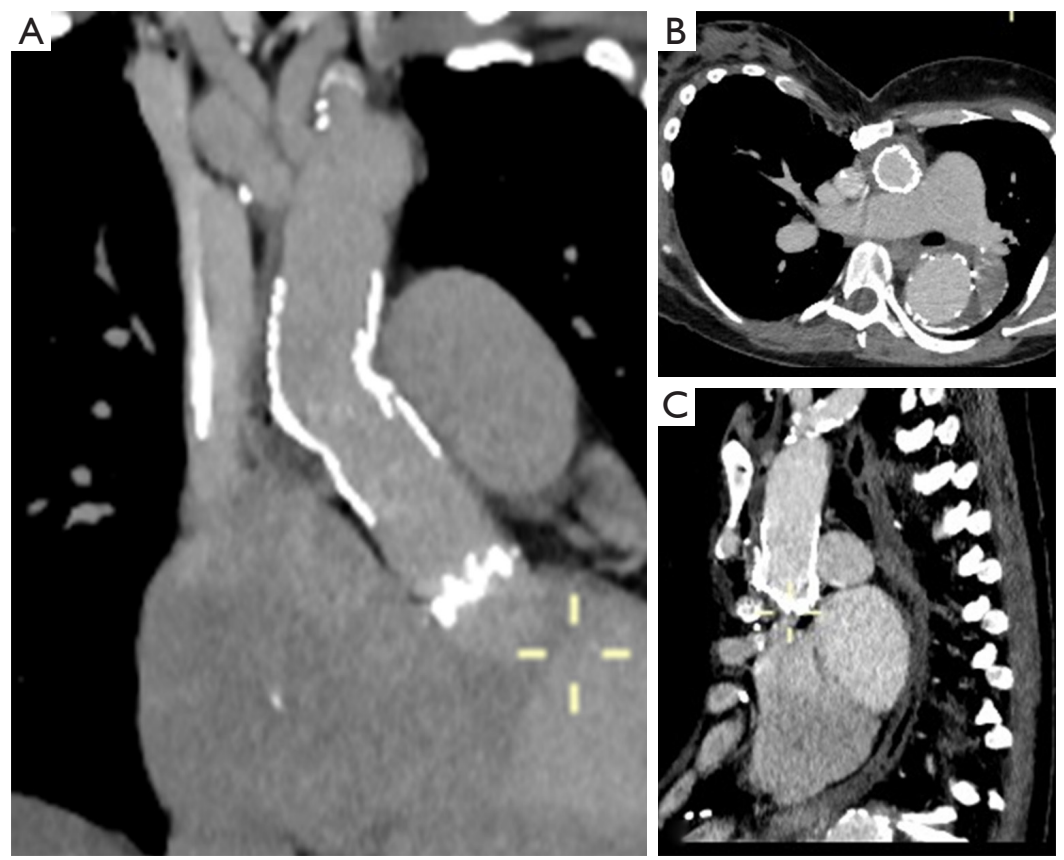

Figure 4 Postoperative CT angiogram at the discharge shows the complete endovascular exclusion of the pseudoaneurysm (A-C). CT, computed tomography.

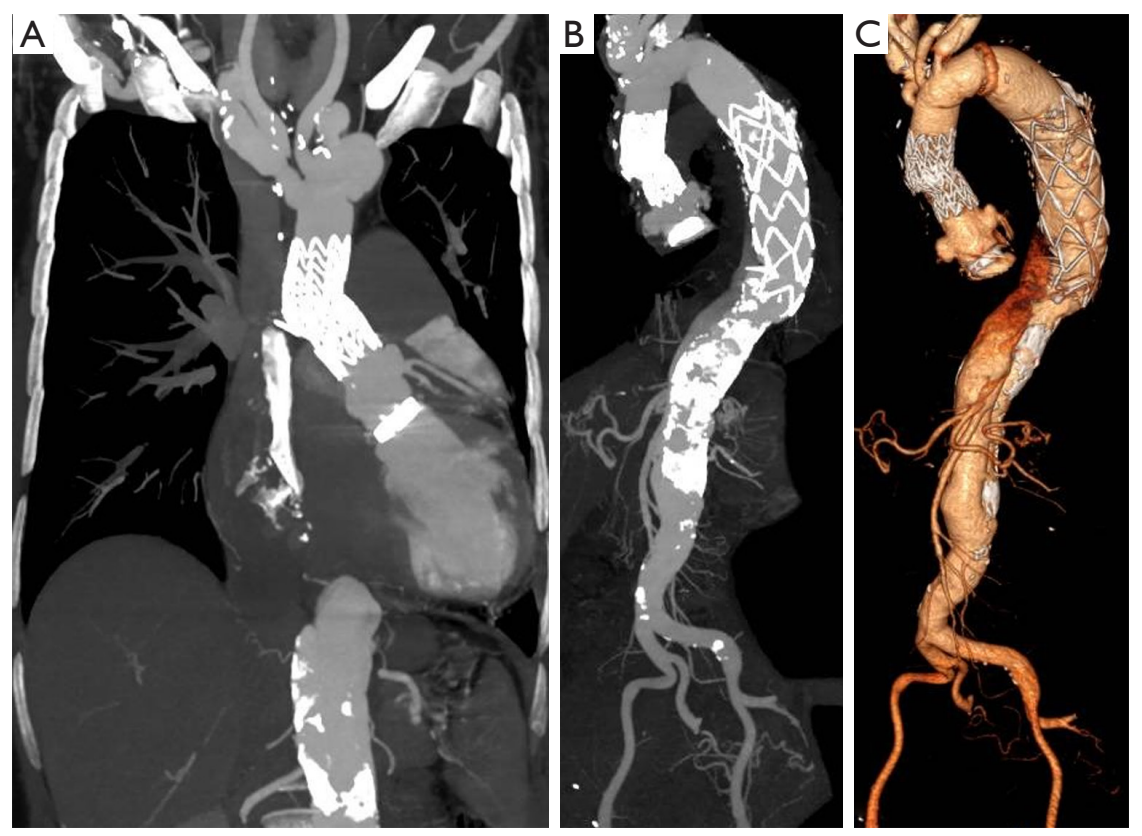

Figure 5 At 2-month follow-up, CT angiogram shows stable endovascular repair of the pseudoaneurysm. CT, computed tomography. 
of ascending aortic pathologies treated with endovascular procedures progressively increased up to the seven cases reported by Preventza et al. in 2014 (8) and even to 21 by Roselli et al. in 2015 (9) and to 15 by Li et al. in 2016 (2). In most of experiences reported in a recent review by Muetterties et al. (5), indications to treatment were type A dissection and pseudoaneurysms and the preferred vascular access was through femoral artery. Thoracic stent grafts were most commonly used to treat type A aortic dissections while abdominal cuffs were primarily used to treat aortic pseudoaneurysms.

In our patient, because of the presence of the mechanical aortic valve prosthesis that required a device with a short tip in order to avoid any contact with the valve itself, we decided to use the left axillary artery as surgical access. Another reason to choose the left axillary artery was that the proximal abdominal cuff is designed with a short delivery sheath that cannot reach the ascending aorta from the femoral artery. This is the reason why several alternative vascular access, such as axillary, carotid and subclavian artery, or even the apex of left ventricle, has begun to be used during endovascular procedure on the ascending aorta (8-12). In our opinion, the approach we used facilitated the accuracy of the deployment allowing to avoid the multiple curvatures of the others, possibles, approaches and increasing the safety and the accuracy of the landing at the coronary ostia. Moreover, in order to have a better proximal and distal sealing, we decided to implant a second abdominal cuff whose positioning was decided considering the echocardiographic probe as reference point and using the angiography to see the exact position of the coronary ostia and the length of the tip as a reference. In order to reduce cardiac output, according to the technique used during transcatheter aortic valve procedure, we decided to position, before the procedure, a temporary transvenous pacing wire into the right ventricle in order to rapidly pace the heart at 120 beats/min and to reduce the arterial blood pressure at $40 \mathrm{mmHg}$ at the moment of cuffs deployment.

The complication most commonly described in patients treated with ascending aorta stent-grafting is endoleak, with an overall rate of $18 \%$ (5). We know that endoleak can increase the risk of aortic rupture, so, aware of this, we thought that this patient, having already replaced the ascending aorta and the aortic valve with a composite valve graft, could be the ideal patient for this procedure. In fact, the presence of a Dacron graft has the advantage to avoid a possible future wall degeneration compared to native aorta.
Beyond the endoleaks, the risk of possible complications such as open conversion, stent graft migration, intraoperative and postoperative myocardial infarction, stroke, device deployment failure or others aorta-related complications, in this type of procedures remains potentially high thus, in the light of this, it is understood that a close monitoring of patients is of primary importance in order to early detect and treat these complication avoiding long-term mortality (5). However, our policy, based on our extensive surgical experience on ascending aorta diseases and on aortic diseases in general, is to reserve an endovascular solution for ascending aorta pathologies only for patients who are considered unfit candidates for open surgery. For this reason we believe that, in selected cases, endovascular treatment of ascending aorta pseudo-aneurysm may be a good option as the case presented in which we considered the endovascular procedure to be safer and more effective than the positioning of a plug or the embolisation of the pseudoaneurysm.

\section{Conclusions}

We presented a case of a patient with an ascending aorta pseudoaneurysm which was successfully treated with an endovascular approach using two abdominal cuffs via a left axillary artery as surgical access. In this case the patients at high surgical risk because of the previous operations and the patient's anatomy was favorable for the use of endovascular technologies. Moreover, we were optimally prepared for a potential conversion since that we performed this case in the hybrid operating room which allows both endovascular and surgical procedures to be performed with the same efficacy and with the same degree of safety. In case of ascending aorta pseudoaneurysms, surgical repair remains the "gold-standard" therapy. This given, in high risk patients, considered unfit for open surgery, endovascular repair could be considered a valid and viable therapeutic option. In order to an optimal outcome and results, an accurate selection of patients is of primary importance and represent the critical point of the decision-making phase. Ascending aorta endovascular repair requires a high degree of technical skill and experience and we strongly suggest to treat this subgroup of patients in high-volume centers in which surgeons and interventional radiologists have a large experience both in surgical and endovascular treatment of the thoracic and thoracoabdominal aorta pathologies in order to be able to deal and treat with any complications immediately. 


\section{Acknowledgements}

None.

\section{Footnote}

Conflicts of Interest: The authors have no conflicts of interest to declare.

Informed Consent: Written informed consent was obtained from the patient for publication of this manuscript and any accompanying images.

\section{References}

1. Kotelis D, Kalder J, Jacobs MJ. Endovascular repair of the ascending aorta: the last frontier? J Thorac Dis 2016;8:E825-6.

2. Li Z, Lu Q, Feng R, et al. Outcomes of Endovascular Repair of Ascending Aortic Dissection in Patients Unsuitable for Direct Surgical Repair. J Am Coll Cardiol 2016;68:1944-54.

3. Kubota H. Endovascular stent graft repair of the ascending aorta-final frontier in the endovascular treatment of the aorta. J Thorac Dis 2016;8:E1358-60.

4. Klonaris C, Georgopoulos S, Katsargyris A. Endovascular treatment of the ascending aorta: new frontiers for thoracic endovascular aneurysm repair? J Thorac Dis 2016;8:1901-3.

doi: 10.21037/jovs.2018.05.15

Cite this article as: Di Marco L, Lovato L, Murana G, Amodio C, Buia F, Di Bartolomeo R, Pacini D. Endovascular repair of ascending aorta pseudoaneurysm. J Vis Surg 2018;4:116.
5. Muetterties CE, Menon R, Wheatley GH 3rd. A systematic review of primary endovascular repair of the ascending aorta. J Vasc Surg 2018;67:332-42.

6 Di Marco L, Lovato L, Murana G, et al. Endovascular stent graft repair of the ascending aorta. Asvide 2018;5:521. Available online: http://www.asvide.com/ article/view/24982

7. Dorros G, Dorros AM, Planton S, et al. Transseptal guidewire stabilization facilitates stent-graft deployment for persistent proximal ascending aortic dissection. J Endovasc Ther 2000; 7:506-12.

8. Preventza O, Henry MJ, Cheong BY, et al. Endovascular repair of the ascending aorta: when and how to implement the current technology. Ann Thorac Surg 2014;97:1555-60.

9. Roselli EE, Idrees J, Greenberg RK, et al. Endovascular stent grafting for ascending aorta repair in high-risk patients. J Thorac Cardiovasc Surg 2015;149:144-51.

10. Saadi EK, Moura Ld, Zago A, et al. Endovascular repair of ascending aorta and coronary stent implantation. Rev Bras Cir Cardiovasc 2011;26:477-80.

11. Mangialardi N, Ronchey S, Malaj A, et al. Case report of an endovascular repair of a residual type A dissection using a not CE not FDA-approved Najuta thoracic stent graft system. Medicine (Baltimore) 2015;94:e436.

12. Kappert U, Ghazy T, Ouda A, et al. Transapical endovascular stenting of penetrating atherosclerotic ulcer of ascending aorta. Ann Thorac Surg 2013;96:e101-3. 